\title{
ON GROUPS OF FIBONACCI TYPE
}

\author{
by A. M. BRUNNER \\ (Received 24th March 1975)
}

\section{An embedding}

Let $w=w\left(a_{0}, a_{1}, \ldots, a_{n-1}\right)$ be a word in the free group freely generated by $a_{0}, a_{1}, \ldots, a_{n-1}$; let $w_{i}$ denote the word $w\left(a_{i}, a_{i+1}, \ldots, a_{i+n-1}\right)$, where the subscripts $j$ in $a_{i}$ are reduced modulo $n$; and let

$$
G(n ; w)=\left\langle a_{0}, a_{1}, \ldots, a_{n-1}: \quad w_{i}=1 ; i=0,1, \ldots, n-1\right\rangle .
$$

Amongst the groups $G(n ; w)$ are those said to be of "Fibonacci type" (see (2)). The Fibonacci groups are the groups $F(r, n)=G(n ; w)$, where $w=$ $a_{0} a_{1} \ldots a_{r-1} a_{r}^{-1}($ see $(3))$.

Now let

$$
E(n ; w)=\left\langle a, b ; \quad b^{n}=1, w\left(a_{0}, a_{1}, \ldots, a_{n-1}\right)=1\right\rangle,
$$

where here $a_{i}$ denotes the element $b^{-i} a b^{i}(0 \leqslant j \leqslant n-1)$. Then, it is a direct consequence of the Reidemeister-Schreier subgroup theorem (see Theorem 2.9 of (4)) that $\boldsymbol{G}(n ; w)$ is embedded as the least normal subgroup of $E(n ; w)$ containing the element $a$; one choses the elements $1, b, \ldots, b^{n-1}$ as Schreier coset representatives.

It is often easier and more instructive to consider $E(n ; w)$ rather than $\boldsymbol{G}(n ; w)$. Apparently this was the case in R. C. Lyndon's proof that $\boldsymbol{F}(r ; n)$ is infinite if $n \geqslant 11$. As $\boldsymbol{G}(n ; w)$ is a normal subgroup of index $n$ in $\boldsymbol{E}(n ; w)$ it is infinite if $E(n ; w)$ is infinite, finite of order $s / n$ if $E(n ; w)$ is finite of order $s$. Also, $G(n ; w)$ inherits subgroup properties. In any case, $E(n ; w)$ is a two-generator two-relator group whose relators can often be easily transformed into simple and workable forms.

This approach may be used to give a simple solution* to part of Problem 3 of (3) (another solution is given in (1)). Indeed, the comments above, together with the argument of Theorem 5 of (3), yield immediately that $F(r ; n)$ is metacyclic of order $r^{n}-1$ when $r \equiv 1$ modulo $n$.

\section{On a conjecture}

In (2), the group $H(r, n, s)=G(n ; w)$, where

$$
w=a_{0} a_{1} \ldots a_{r-1}\left(a_{r} a_{r+1} \ldots a_{r+s-1}\right)^{-1},
$$

* I understand from the referee, that M. J. Dunwoody has preceded me with this observation, which was made at the International Conference of Mathematicians, Vancouver, 1974. 
is defined. According to $\S 1$, the group $H(r, n, s)$ is embedded as a normal subgroup of index $n$ in the group $E(n ; w)=\left\langle c, b ; b^{n}=1, b^{r} c^{r}=c^{s} b^{s}\right\rangle$, where $c=a b^{-1}$.

C. M. Campbell and E. F. Robertson conjecture (in (2)) that $\boldsymbol{H}(r, 4,2)$ is metacyclic if $r$ is odd. This is so; in fact, it will follow below that $\boldsymbol{H}(r, 4,2)$ is metacyclic of order $(r-2)\left(2^{\xi}-2.2^{\eta}+2\right) / 2$, where $\xi \equiv 4 \alpha, \eta \equiv 2 \alpha$ reduced modulo $4(r-2)$, with $\alpha$ given by $4 \alpha \equiv 1$ modulo $r-2$.

Consider $E(4 ; w)=\left\langle c, b ; b^{4}=1, b^{r} c^{r}=c^{2} b^{2}\right\rangle$. If $r$ is even then $E(4 ; w)$ is infinite, as it has the group $\left\langle b, c ; b^{2}=c^{2}=1\right\rangle$ as an epimorph. Thus we assume in the sequel that $r$ is odd.

In case $r \equiv 3$ modulo 4 , place $d=b c^{r-2}$, and in case $r \equiv 1$ modulo 4 , place $d=c^{r-2} b^{-1}$. Then,

$$
E(4 ; w)=\left\langle d, c ; d d_{r-2}=d_{r}\left(d c^{-(r-2)}\right)^{4}=1\right\rangle,
$$

where $d_{i}$ is used to denote $d^{c^{i}}=c^{-i} d c^{i}$ for integers $i$.

Our first observation is that $\left(d c^{-(r-2)}\right)^{4}=1$ can be written in the form $c^{4(r-2)}=d d_{r-2}\left(d d_{r-2}\right)^{c^{2(r-2)}}$; and therefore, since $d_{r}=d d_{r-2}$, in the form

$$
c^{4(r-2)}=d d_{2(r-2)}
$$

The relation $d d_{r-2}=d_{r}$ is the same as $d=d_{r-2}^{c^{2}} d_{r-2}^{-1}$; hence, using (i), we have $d_{r-2}=d_{2(r-2)}^{c^{2}} d_{2(r-2)}^{-1}=d_{2}^{-1} c^{4(r-2)} c^{-4(r-2)} d$, and so

$$
d_{r-2}=d_{2}^{-1} d \text {. }
$$

From (i), we have $c^{4(r-2)}=d_{r} d_{r-2}^{-1} d_{2(r-2)}=d_{2} d^{-1} d_{r-2}$. In particular, $d_{2} d^{-1} d_{r-2}=d_{4} d_{2}^{-1} d_{r}=d_{4} d_{2}^{-1} d d_{r-2}$, so that $d_{4}=d_{2} d^{-2} d_{2}$. Thus, on the one hand, by (ii) we have $d_{r}=d_{4}^{-1} d_{2}=d_{2}^{-1} d^{2}$, and on the other, $d_{r}=d d_{r-2}=$ $d d_{2}^{-1} d$. We conclude that $d_{2} d=d d_{2}$.

Relation (ii) implies $d_{2(r-2)}=d_{r}^{-1} d_{r-2}=d_{2}^{-1} d_{4} d_{2}^{-1} d=d^{-1}$, since $d_{4}=$ $d_{2} d^{-2} d_{2}$; whence, by (i) we have $c^{4(r-2)}=1$. Moreover, since $d_{r}=d_{2}^{-1} d^{2}$, we obtain $d_{r+2}=d_{4}^{-1} d_{2}^{2}=d^{2}$.

Conversely, the relations $d_{r+2}=d^{2}, d_{4}=d_{2}^{2} d^{-2}$ and $c^{4(r-2)}=1$ imply the original two. Firstly, since $(4(r-2),(r+2))=1$ we obtain, using $c^{4(r-2)}=1$ and $d_{r+2}=d^{2}$, that $d_{1}=d^{\mu}$ for some integer $\mu$, and hence that $d_{i} d_{j}=d_{i} d_{i}$ for each integer $i$ and $j$. Also, $d_{r+2}=d^{2}$ implies $d_{r}=d_{-2}^{2}, d_{r-2}=d_{-4}^{2}$; and as $d=d_{-2}^{2} d_{-4}^{-2}$, we conclude that $d_{r}=d d_{r-2}$. Further, the relation $d_{4}=d^{2} d_{2}^{-2}$ implies that $d_{6}=d_{2}^{2} d^{-4}$ and $d_{8}=d^{-4}$; so, as $d_{r-2}=d_{-4}^{2}$, we have that $d_{2(r-2)}=$ $d_{-8}^{4}=d^{-1}$, and this verifies relation (i). It follows that

$$
E(4 ; w)=\left\langle d, c ; d_{r+2}=d^{2}, d_{4}=d_{2}^{2} d^{-2}, c^{4(r-2)}=1\right\rangle .
$$

Now let $\alpha$ be given by $\alpha(r+2) \equiv 1$ modulo $4(r-2)$ (equivalently by 
$4 \alpha \equiv 1$ modulo $r-2$ ). Then, if $e=c^{r+2}$, we have $c=e^{\alpha}$; consequently $d^{e}=d^{2}$, and so $d_{4}=d_{2}^{2} d^{-2}$ becomes $d^{2 \xi-22^{\eta}+2}=1$, where $\xi$ and $\eta$ are given by $4 \alpha$ and $2 \alpha$, respectively, when these are reduced modulo $4(r-2)$. Since $d^{e}=d^{2}$, we conclude that $d^{k}=1$. Thus

$$
E(4 ; w)=\left\langle d, e ; d^{e}=d^{2}, d^{k}=1, e^{4(r-2)}=1\right\rangle .
$$

\section{REFERENCES}

(1) C. M. CAMPBELL and E. F. RoberTSON, On metacyclic Fibonacci groups, Proc. Edinburgh Math. Soc. 19 (1975), 253-256.

(2) C. M. CAMPBELL and E. F. RoBerTSON, On a class of finitely presented groups of Fibonacci type, J. London Math. Soc. 11 (1975), 249-255.

(3) D. L. Johnson, J. W. WamsLeY and D. WRight, The Fibonacci groups, Proc. London Math. Soc. (3) 29 (1974), 577-592.

(4) W. Magnus, A. KarRass and D. Solitar, Combinational group theory (Interscience (John Wiley \& Sons), New York, London, Sydney, 1966).

9 Cambridge Street

BRIGHTON

VICTORIA 3187

Australia 